\title{
AVALIAÇÃO DO NÍVEL DE ESTRESSE FíSICO E PSICOLÓGICO DE GESTANTES PARTICIPANTES DE UM PROJETO DE EXTENSÃO
}

Camila Balsamo Gardim; Rayana Loch Gomes; Marjory Mineko Washio; Roselene Modolo Regueiro Lorençoni.

Campus de Presidente Prudente - Faculdade de Ciências e Tecnologia - Fisioterapia. E-mail: camila_gardim@hotmail.com

\section{RESUMO}

Introdução: O estresse e a produtividade relacionam-se diretamente, pois ele induz a produção de adrenalina, e sem este hormônio, o indivíduo torna-se improdutivo. Durante a gravidez, o corpo da mulher sofre diversas alterações físicas e psicológicas. Por isso é considerado um período de estresse emocional muito intenso. Objetivo: Verificar a presença do nível de estresse físico e/ou psicológico de gestantes antes e após realizarem exercícios aquáticos. Metodologia: Foram avaliadas 7 gestantes antes e após protocolo de exercícios aquáticos. Essas gestantes responderam a um questionário que avalia o nível de estresse físico e psicológico (Lipp), que por meio deste pode-se obter os resultados referentes ao estudo. Resultados: Não houve diferença estatisticamente significante entre o nível de estresse pré e pós intervenção. Conclusão: Os níveis de estresse físico e psicológico permaneceram o mesmo antes e após os exercícios aquáticos.

Palavras-chave: estresse; gestantes; exercício, estresse físico e estresse psicológico

\section{INTRODUÇÃO}

Em 1936, Hans Selye, utilizou o termo "estresse" no campo da saúde para designar a resposta do organismo frente a um estressor. É um processo complexo que envolve aspectos bioquímicos, físicos e psicológicos. As queixas mais comuns que as pessoas costumam relatar são fadiga, hipertensão, desânimo e falta de apetite. Porém, a resposta para cada ser humano é diferente, pois depende do estilo de vida de cada pessoa e da sua habilidade de lidar com situações estressantes. Contudo, Selye também enfatiza que o estresse faz parte do cotidiano, pois o ser humano está sujeito a mudanças em diferentes setores de sua vida ${ }^{1-4}$.

O termo "Síndrome Geral da Adaptação" refere-se a uma série de alterações que o organismo sofre quando este passa por uma quebra de homeostase interna, ou seja, quando o organismo se torna incapaz de manter sua constância. O modelo trifásico, proposto por Selye (1936), compreende as seguintes fases: Fase de alarme, Fase de resistência e Fase de exaustão ${ }^{5}$.

A primeira é a fase em que o Sistema Nervoso Central percebe a situação de tensão, e começa a preparar o sistema de defesa do organismo contra o agente estressor. Já na segunda, se o agente estressor perdura por um período prolongado, o organismo aumenta sua capacidade de resistência, e há adaptação ao estressor. Porém, se há insistência do estresse, o nível de 
resistência diminui e inicia-se a fase de exaustão, onde alguns sintomas da primeira fase acentuam-se e outros novos desenvolvem-se. Os mecanismos responsáveis pela homeostase se tornam insuficientes caso os efeitos do estressor permanecer por muito tempo, fazendo com que a energia adaptativa esgote-se ${ }^{2,6}$.

Lipp ${ }^{7}$, em um estudo posterior, identificou uma quarta fase, denominada "quase exaustão", situada entre a segunda e a terceira fase. Nessa nova fase, o indivíduo é caracterizado por um enfraquecimento, pois não consegue mais adaptar-se ou resistir ao estressor, tornando-se suscetível ao aparecimento de doenças não tão graves quanto as da fase final, mas significativas $2,5,6,8$

O estresse e a produtividade relacionam-se diretamente, pois ele induz a produção de adrenalina, e sem este hormônio, o indivíduo torna-se improdutivo. Mas o estresse em excesso faz com que esse rendimento caia e a suscetibilidade a contrair doenças aumente ${ }^{2,6}$.

Durante a gravidez, o corpo da mulher sofre diversas alterações físicas e psicológicas. Por isso é considerado um período de estresse emocional muito intenso. O corpo está em constante transformação, há sentimento de medo e ansiedade pelo parto, a insegurança em relação à vida conjugal e em iniciar o papel de "mãe" e cuidadora e questões relacionadas a dificuldades financeiras. Mas vale ressaltar que cada indivíduo reage diferentemente a esses agentes estressores. O grau de resposta de estresse depende de fatores genéticos, características de personalidade, experiência anterior e a forma de lidar com o estresse ${ }^{9-12}$.

A exposição materna à adrenalina, noradrenalina e cortisol gerados pelo estresse pode ser prejudicial à saúde da mãe e do feto. A gestante pode ser candidata à depressão pós-parto (DPP) e à ocorrência de aborto espontâneo, hipertensão arterial induzida pela gravidez, ansiedade, além de manifestações alérgicas como asma, urticária, eczema. E o bebê pode apresentar problemas temperamentais, prematuridade abaixo do peso ${ }^{12,13}$.

A prática regular de exercícios físicos pode ocasionar a redução dos níveis dos hormônios estressantes citados. Além de proporcionar diversos outros benefícios, como o aumento da autoestima, diminuição da depressão e ansiedade, diminuição da freqüência cardíaca, melhor disposição física, o que contribui para o pós-parto, quando a mãe for segurar seu bebê no colo. Além de auxiliar na distração e relaxamento, no controle do ganho de peso excessivo e atenuar os desconfortos físicos comuns da gestação ${ }^{14-16}$. 


\section{OBJETIVO}

O objetivo desse estudo foi verificar a presença do nível de estresse físico e/ou psicológico de gestantes antes e após realizarem exercícios aquáticos.

\section{METODOLOGIA}

\section{Natureza da Pesquisa e Casuística}

A casuística do estudo foi composta por 7 gestantes com média de idade de 28,5 anos. Duas começaram o protocolo aos dois meses de gestação, uma no terceiro mês, uma no quinto mês e três aos 7 meses de gestação. As mesmas foram esclarecidas sobre o objetivo e os procedimentos do estudo. As gestantes que concordaram em participar assinaram o "Termo de Consentimento Livre e Esclarecido".

\section{Desenho do estudo}

Para a realização das coletas de dados, as gestantes foram entrevistadas por uma equipe treinada. Inicialmente, foram explicados os objetivos do estudo e as voluntárias que concordaram em participar do estudo, assinaram o termo de consentimento livre e esclarecido (protocolo número 81/2011). Após essa etapa, os indivíduos responderam em forma de entrevista o Lipp.

\section{Coleta de dados}

A aplicação dos questionários foi realizada de forma padronizada e os voluntários foram orientados a questionar acerca de dúvidas que surgiram durante a aplicação. O questionário Lipp compreende de um primeiro quadro (Fase de Alerta), composto de 12 itens referente a sintomas físicos e 03 psicológicos que a pessoa tenha experimentado nas últimas 24 horas. 0 segundo quadro (Fase de Resistência e Quase-Exaustão) composto de 10 sintomas físicos e 05 psicológicos e está relacionado aos sintomas experimentados na última semana. E o terceiro quadro (Fase de Exaustão), composto de 12 sintomas físicos e 11 psicológicos, refere-se a sintomas experimentados no último mês. Alguns dos sintomas que aparecem no quadro 1 voltam a aparecer no quadro 3, mas com intensidade diferente ${ }^{17}$. Esse questionário foi aplicado a gestante logo após a aferição dos parâmetros vitais, antes delas entrarem na água, e reaplicado logo após a saída da piscina. 


\section{Protocolo de exercícios aquáticos}

Antes e após as sessões foram verificados os sinais vitais de frequência cardíaca, pressão arterial, saturação de oxigênio e aplicação da Escala de Borg para verificação de sensação de falta de ar e desconforto musculares em MMII.

O programa de exercícios físicos aquáticos foi realizado em uma piscina coberta e aquecida com temperatura entre $30^{\circ} \mathrm{C}$ e $34^{\circ} \mathrm{C}$, 2 vezes por semana, com duração de 50 minutos, grupos de no máximo 5 gestantes.

O protocolo foi dividido em fases de alongamento, aquecimento, exercícios lúdicos e de fortalecimento muscular, mobilizações articulares, analgesia e relaxamento.

- Aquecimento: 5 a 10 minutos - deambular na piscina lateralmente, de costas e de frente e pedalar ao redor da piscina sentada no espaguete.

- Alongamento: 10 minutos (12 a 16 segundos cada exercício)

- Região cervical: inclinação da cabeça para direita e para esquerda, flexão e extensão da cabeça, rotação da cabeça, movimentos circulares com a cabeça

- Membros Superiores (MMSS): flexão do ombro e do cotovelo, extensão do cotovelo e flexão a 90 do ombro, alongamento dos extensores e flexores do braço, flexão do ombro a 90ㅇ e adução até o limite da amplitude.

- Membros Inferiores (MMII): flexão de joelho, extensão de quadril com a perna da frente dobrada e a de trás esticada, abertura lateral das pernas.

- Fortalecimento (2 séries de 10 a 15 repetições): 20 minutos

- MMSS

1. Bíceps braquial: em pé, com as costas retas, os pés afastados e manter os joelhos semiflexionados. Com os braços estendidos ao longo do corpo, segurar o halter. Com as mãos voltadas para o corpo, dobrar os braços até a altura dos ombros, contra a resistência da água. Voltar a posição da água.

2. Abdutores: em pé, com o quadril em retroversão, afastar os pés mantendo os joelhos semiflexionados. Com os braços elevados a 90 e braços estendidos, segurando um halter. Abaixar os braços até encostar-se ao corpo e elevá-los lentamente, contra a resistência da água

3. Com os braços aduzidos a 90 ㅇ e braços estendidos, segurando os halteres, levar os braços em direção aos outros, cruzando-os a frente do corpo e voltar para a posição inicial

Abdominais e diminuição da diástase 
1. Contração isométrica do abdome

2. Reto abdominal: apoiada em boias espaguetes e com os pés fixados na barra da parede. Contrair o abdominal para levar o tronco em direção a parede e retornar à posição inicial

3. Oblíquos: apoiada em boias espaguetes e com as pernas estendidas, flexioná-las e rodar o quadril para um lado e depois para o outro

4. Abdominal inferior: apoiada em boias espaguetes, com as pernas estendidas, fazer uma abdução seguida de uma adução, cruzando as duas pernas

$\underline{- \text { MMII }}$

1. Adutores, abdutores e extensores: encaixar o halter redondo em um pé e realizar a abdução, adução e extensão da perna, lutando contra a força da água

2. Quadríceps: flexionar o joelho e o quadril a 90ํ, realizar extensão da perna Assoalho pélvico

3. Contração isométrica da musculatura pélvica com contração de abdominais

- Relaxamento: 10 minutos (colocar o colete)

As gestantes permaneceram em decúbito dorsal com flutuadores, o terapeuta realizou movimentos com o corpo da gestante, de maneira suave e relaxante.

O ambiente permaneceu com baixa luminosidade e com música relaxante. Nesse momento, o terapeuta também pode optar por colocar a gestante sentada em uma plataforma na piscina, caso julgue necessário em relaxamento com mobilizações, solturas e massagem.

É importante ressaltar alguns cuidados durante os exercícios, como manter uma boa postura ao executar os exercícios. As séries poderão ser realizadas com intensidade de leve a moderada.

\section{Análise dos dados}

A caracterização da amostra foi realizada descritivamente (média, desvio padrão, frequência e porcentagem) A normalidade dos dados foi testada por meio do teste Shapiro-Wilk. A comparação dos momentos pré e pós-intervenção do questionário LIPP foi realizada por meio do teste $\mathrm{t}$ de Student. Os testes estatísticos foram aplicados por meio do softwareGraphPad (5.0, Prisma ${ }^{\oplus}$ ) e padronizou-se o nível de significância de $p<0,05$. 


\section{RESULTADOS}

Na tabela 1 está representado a caracterização da amostra, com um total de 7 gestantes, no momento pré e pós intervenção, sendo que os dados estão dados em números absolutos, porcentagem, média e desvio-padrão. Já a tabela 2, apresenta a comparação dos valores encontrados nas três fases do Questionário LIPP pré e pós intervenção, também realizado em 7 gestantes.

Tabela 1: Caracterização da amostra de gestantes pela idade, mês gestacional e vínculo empregatício.

\begin{tabular}{lcc}
\hline \multicolumn{1}{c}{ Variáveis } & Gestantes $(\mathbf{n}=\mathbf{7})$ \\
\hline Idade (anos) & & $28,57 \pm 4,03$ \\
Mês gestacional & 2o & $2(28,56 \%)$ \\
& 3o & $1(14,28 \%)$ \\
& 5o & $1(14,28 \%)$ \\
Trabalha & 7o & $3(42,88 \%)$ \\
& 9o & - \\
& SIM & $3(42,88 \%)$ \\
& NÃO & $4(47,12 \%)$
\end{tabular}

*A variável idade foi expressa em média e desvio padrão e as variáveis mês gestacional e trabalho em frequência e porcentagem.

Tabela 2: Comparação dos valores encontrados no Questionário LIPP pré e pós intervenção com exercícios aquáticos.

\begin{tabular}{lccc}
\hline Questionário LIPP & $\begin{array}{c}\text { Pré Intervenção } \\
(\mathbf{n}=7)\end{array}$ & $\begin{array}{c}\text { Pós Intervenção } \\
(\mathbf{n}=7)\end{array}$ & Valores de $\boldsymbol{p}$ \\
\hline Fase 1 - Alerta (alarme) & $4,28 \pm 0,51$ & $4,29 \pm 0,52$ & $p=0,7088$ \\
Fase 2 - Resistência (luta) & $7 \pm 0,52$ & $6,28 \pm 0,52$ & $p=0,8809$ \\
$\begin{array}{l}\text { Fase 3 - Exaustão } \\
\text { (esgotamento) }\end{array}$ & $0,48 \pm 0,50$ & $0,36 \pm 0,50$ & $p=0,4680$ \\
\hline média \pm desvio-padrão & & &
\end{tabular}

\section{DISCUSSÃO}

Neste estudo, três das sete gestantes apresentaram redução do nível de estresse pósintervenção aquática (fase de resistência e fase de exaustão), mas, no geral, não houve diferença estatisticamente significante nos valores de p. Esses resultados podem ser explicados, pois não 
houve um acompanhamento periódico dessas gestantes, sendo que a aplicação do questionário foi realizada apenas uma vez. Fato importante, visto que, em apenas um único dia de entrevista, já foi relatado uma melhora no nível de estresse desses indivíduos. Dessa forma, é valido que haja uma verificação mais branda, para que possam ser obtidos melhores resultados na amostra.

Satyapriya et al. ${ }^{18}$ utilizaram práticas de Yoga para analisar seus efeitos sobre o estresse percebido em gestantes saudáveis. No estudo 90 mulheres foram subdivididas em 2 grupos: controle e intervenção. No grupo intervenção foram utilizadas posturas de Yoga, técnicas respiratórias e técnicas de relaxamento específicas para dada trimestre da gestação. $E$ foi verificado que a prática de Yoga promoveu a redução do estresse percebido (31,6\%) o que não ocorreu no grupo controle $(6,6 \%)$.

Cruz e Notini ${ }^{19}$ introduziram atividades recreativas durante aulas de hidroginástica para a verificação do nível de estresse em 10 gestantes. Inicialmente foi verificado que $20 \%$ das gestantes apresentavam baixo nível de estresse, $70 \%$ nível médio e 10\% nível alto de estresse. Após 4 meses de intervenção contatou-se que $60 \%$ das alunas apresentavam baixo nível de estresse, $40 \%$ nível médico e o grau máximo não foi detectado. Portanto, os resultados demonstram a redução do estresse após a intervenção proposta.

\section{CONCLUSÃO}

De acordo com os resultados, os níveis de estresse físico e psicológico permaneceram o mesmo antes e após os exercícios aquáticos. Entretanto, devem-se evidenciar algumas limitações do presente estudo como a existência de uma amostra pequena e a ausência de um grupo controle. . Estudos futuros devem ser realizados com uma amostra maior, um maior número de sessões e, se disponível, um instrumento específico para verificar estresse em gestantes.

\section{REFERÊNCIAS}

1. Guerrer FJL, Bianchi ERF. Caracterização do estresse nos enfermeiros de unidade de terapia intensiva. Rev. Esc. Enferm. USP. 2008; 42(2): 355-362. http://dx.doi.org/10.1590/S0080$\underline{62342008000200020}$

2. Pafaro RC, Martino MMF. Estudo do estresse do enfermeiro com dupla jornada de trabalho em um hospital de oncologia pediátrica de Campinas. Rev. Esc. Enferm. USP. 2003; 38(2): 152-160. http://dx.doi.org/10.1590/S0080-62342004000200005

3. Murta SG, Tróccoli BT. Avaliação de intervenção em estresse ocupacional. Psic.: Teor. e Pesq. 2004; 20(1): 39-47. 
4. Margis R, Picon P, Cosnner AF, Silveira RO. Relação entre estressores, estresse e ansiedade. R. Psiquiatr. RS. 2003; 25(1): 65-74.

5. Santos FD, Cunha MHF, Robazzi MRC, Pedrão L, Silva LA, Terra CS. O estresse do enfermeiro nas unidades de terapia intensiva adulto: uma revisão da literatura. Rev. Eletrônica Saúde Mental Álcool Drog. 2009; 6(1): 1-16.

6. Goulart Junior E, Lipp MEN. Estresse entre professoras do ensino fundamental de escolas públicas estaduais. Psicol. estud. 2008; 13(4): 847-857. http://dx.doi.org/10.1590/S1413$\underline{73722008000400023}$

7. Lipp MEN. Manual do Inventário de Sintomas de Stress para adultos de Lipp (ISSL). Casa do Psicólogo, São Paulo, 2000.

8. Santos AFO, Cardoso CL. Profissionais de saúde mental: estresse e estressores ocupacionais stress e estressores ocupacionais em saúde mental. Psicol. Estud. 2010; 15(2): 245-253. http://dx.doi.org/10.1590/S1413-73722010000200003

9. Mulder EJ, Robles de Medina PG, Huizink AC, Van den Bergh BR, Buitelaar JK, Visser GH. Prenatal maternal stress: effects on pregnancy and the (unborn) child. Early. Hum. Dev. 2002; 70(3): 3-14. http://dx.doi.org/10.1016/S0378-3782(02)00075-0

10. Piccinini CA, Sobreira LR, Lopes AG, Nardi T. Gestação e a constituição da maternidade. Psicol. Estud. 2008; 13(1): 63-72. http://dx.doi.org/10.1590/S1413-73722008000100008

11. Correia DS, Santos LVA, Calheiros AMN, Vieira MJ. Adolescentes grávidas: sinais, sintomas, intercorrências e presença de estresse. Rev. Gaúcha. Enferm. 2011; 32(1): 40-47. http://dx.doi.org/10.1590/S1983-14472011000100005

12. Rodrigues OMPR, Schiavo RA. Stress na gestação e no puerpério: uma correlação com a depressão pós-parto. Rev. Bras. Ginecol .Obstet. 2011; 33(9): 252-257. http://dx.doi.org/10.1590/S0100-72032011000900006

13. Chrousos GP. Stress and disorders os the stress system. Nat. Rev. Endocrinol. 2009; 5: 374381. http://dx.doi.org/10.1038/nrendo.2009.106

14. Martins CO, Jesus JF. Estresse. Exercício Físico, Ergonomia e Computador. Rev. Bras. Cienc. Esp. 1999; 21(1): 807-813.

15. Lima FR, Oliveira N. Gravidez e exercício. Rev. Bras. Reumatol. 2005; 45(3): 188-190. http://dx.doi.org/10.1590/S0482-50042005000300018

16. Segato L, Andrade A, Vanconcellos DIC, Matias TS, Rolin MKSB. Ocorrência e controle do estresse em gestantes sedentárias e fisicamente ativas. R. da Educação Física. 2009; 20(1): 121129.

17. Lipp MEN; Guevara AJH. Validação Empírica do Inventário de Sintomas de Stress (ISS). Estudos Psicolog. 1994; 11(3): 43-49. 
18. Satyapriya M, Nagendra HR, Nagarathna R, Padmalatha V. Effect of integrated yoga on stress and heart rate variability in pregnant women. Int. J. Gynaecol. Obstet. 2009; 4(3): 218-222. http://dx.doi.org/10.1016/i.ijgo.2008.11.013

19. Cruz HR, Notini JR. Alterações nos níveis de stress em mulheres adultas a partir da introdução de atividades recreativas nas aulas de hidroginástica. Facul. Integradas Maria Thereza. 2004; 3: 123. 